\title{
Metabolism of Fatty Acids by Bovine Spermatozoa
}

\author{
By A. R. NEILL \\ Animal Research Institute, Yeerongpilly, Brisbane, Queensl. 4105, Australia \\ and C. J. MASTERS \\ Department of Biochemistry, University of Queensland, St. Lucia, Brisbane, Queensl. 4067, Australia
}

(Received 17 December 1971)

\begin{abstract}
The incorporation of ${ }^{14} \mathrm{C}$-labelled myristic, palmitic, stearic, oleic and linoleic acids in vitro into the lipids of bovine spermatozoa was measured at intervals from $2 \mathrm{~min}$ to $2 \mathrm{~h}$. All acids were rapidly incorporated into diglycerides, myristic acid being metabolized to the greatest extent. Whereas the low incorporation of acids into total phospholipids reflected the relative stability of the major phospholipid fractions in sperm, the minor phospholipids, particularly phosphatidylinositol, showed comparatively high metabolic activity. Although, in general, saturated acids were incorporated more actively than unsaturated substrates, stearic acid was poorly incorporated into all lipids except phosphatidylinositol. In regard to fatty acid composition of sperm lipids it was notable that diglycerides contained myristic acid as the major component, and this acid was also a prominent moiety of phosphatidylinositol. Docosahexaenoic acid was the principal fatty acid of the major phospholipid classes. These findings have been discussed in relation to the role of lipids in the metabolism of spermatozoa.
\end{abstract}

Fatty acids appear to play an important role in the metabolism of spermatozoa. Endogenous fatty acids derived from plasmalogenic phospholipids, for example, have been shown to support the respiration of ovine spermatozoa in the absence of glycolytic substrates (Hartree \& Mann, 1961), and exogenous fatty acids are also oxidized by sperm (Flispe, 1960; Mills \& Scott, 1968; Payne \& Masters, 1970). Of greater metabolic significance than this latter process, however, may be the active incorporation of these acids into the acyl moieties of spermatozoal lipids. Whereas diglycerides incorporate long-chain fatty acids most actively, it has been suggested that phospholipids play a major role in the initial uptake of these components (Payne \& Masters, 1970).

The present study was undertaken to enable a definition and evaluation of the rate of incorporation of fatty acids into the individual lipids of bovine spermatozoa. Of the phospholipid components, phosphatidylinositol was found to have an especially high incorporation, and the significance of this finding has been discussed in relation to the composition and metabolism of spermatozoal lipids. Some of the work included in this paper has been reported in brief by Neill \& Masters (1971).

\section{Experimental}

\section{Materials}

$\left[1-{ }^{14} \mathrm{C}\right]-M y r i s t i c,-s t e a r i c,-o l e i c,-$ linoleic acids and [U-14 C]palmitic acid were obtained from The Radiochemical Centre, Amersham, Bucks., U.K. Their purity was at least $98 \%$ as measured by thin-layer and gas-liquid chromatography. Albumin (bovine, fatty acid poor) was a product of Calbiochem, Los Angeles, Calif., U.S.A. Standard phospholipids were purchased from Applied Science Laboratories, State College, Pa., U.S.A. and Pierce Chemicals Company, Rockford, Ill., U.S.A. Silica gel HR and silica gel G were the products of Merck A.-G., Darmstadt, Germany. Analytical-grade reagents were used in all experiments, and solvents were dried and freshly distilled before use.

\section{Methods}

Incubations. Samples $(1 \mathrm{ml})$ of freshly ejaculated bovine semen $\left(1.4 \times 10^{9}\right.$ to $1.6 \times 10^{9}$ spermatozoa $\left./ \mathrm{ml}\right)$ were incubated under aerobic conditions at $32^{\circ} \mathrm{C}$ with $1 \mathrm{ml}$ of Krebs-Ringer phosphate buffer, $\mathrm{pH} 7.4\left(\mathrm{Ca}^{2+}\right.$ free; Umbreit et al., 1964) containing 3\% (w/v) bovine serum albumin, $0.1 \mathrm{ml}(0.5 \mu \mathrm{Ci})$ of ${ }^{14} \mathrm{C}$ labelled fatty acid solubilized in Krebs-Ringer phosphate albumin buffer, pH7.4, by the method of Payne \& Masters (1969), $0.1 \mathrm{ml}$ of $4 \%(w / v)$ fructose solution and 300 units of penicillin. Incubations were done in air in a Grant shaking water bath (120 strokes/min) for periods of $2 \mathrm{~min}, 15 \mathrm{~min}, 30 \mathrm{~min}, 1 \mathrm{~h}$ and $2 \mathrm{~h}$.

Lipid extraction. At the end of each incubation period $19 \mathrm{vol}$. of chloroform-methanol $(2: 1, \mathrm{v} / \mathrm{v})$ was added to the flasks, and the lipids were extracted and washed by the procedure of Folch et al. (1957). Extracting solvent was removed by rotary film

Vol. 127 
evaporation and the residue was dried in vacuo over $\mathbf{P}_{2} \mathrm{O}_{5}$. Dried samples were stored in chloroformmethanol $(5: 1, \mathrm{v} / \mathrm{v})$ at $-40^{\circ} \mathrm{C}$.

Where spermatozoal and seminal plasma lipids were examined separately, the two components of semen were separated by centrifugation at $450 \mathrm{~g}$ for $20 \mathrm{~min}$. The cells were washed several times with ice-cold $0.9 \%(\mathrm{w} / \mathrm{v}) \mathrm{NaCl}$ and the washings were combined with the plasma fraction. Lipids were then extracted from the separated components as above.

Lipid fractionation. Neutral lipids were separated by t.l.c. on $250 \mu \mathrm{m}$ thick layers of silica gel $\mathrm{G}$ that had been activated at $110^{\circ} \mathrm{C}$ for $30 \mathrm{~min}$. Hexane-diethyl ether-acetic acid (80:20:1, by vol.) was used as developing solvent.

Phospholipids were separated by two-dimensional t.l.c. on $500 \mu \mathrm{m}$ layers of silica gel HR $(20 \mathrm{~cm} \times 20 \mathrm{~cm}$ plates) activated at $110^{\circ} \mathrm{C}$ for $1 \mathrm{~h}$, by using a modification of the method of Owens (1965). Plates were developed in chloroform-methanol-water-acetic acid (65:43:3:1, by vol.) in the first dimension to within $4 \mathrm{~cm}$ of the top of the plate. Solvent was removed under a stream of dry $\mathrm{N}_{2}$, and the plates were developed completely in the same dimension by using hexane-diethyl ether-acetic acid $(80: 20: 1$, by vol.) in order to remove neutral lipid to the top of each plate. Solvent was again removed under dry $\mathrm{N}_{2}$ and the lipid track was exposed to $\mathrm{HCl}$ fumes to hydrolyse plasmalogens as described by Viswanathan et al. (1968). Excess of $\mathrm{HCl}$ was removed by exposing the plates to a stream of dry $\mathrm{N}_{2}$ for $1 \mathrm{~h}$. The plates were then developed in the second dimension by using chloroform-methanol-water $(60: 35: 8$, by vol.) as developing solvent.

Radioactivities. Thin-layer plates were exposed to X-ray film (Kodirex No-screen, Kodak Aust. Pty. Ltd.). After development, areas of activity were identified and the silica gel containing the labelled lipids was scraped into vials containing $4 \mathrm{ml}$ of scintillator fluid [0.4\% 2,5-diphenyloxazole, $0.01 \%$ 1,4-bis-(5-phenyloxazol-2-yl)benzene in toluene]. Radioactivity was measured by using a NuclearChicago liquid-scintillation counter, the counting efficiency being measured by use of an external standard. Samples of the total activities incubated and extracted were counted under similar conditions.

The identity of the various labelled lipid components was established from the comparative behaviour of standard phospholipids under various chromatographic conditions.

Phosphorus analyses. Lipid extracts from washed bovine spermatozoa, containing up to $25 \mu \mathrm{g}$ of $\mathrm{P}$, were separated by the two-dimensional t.l.c. procedure described previously. After development, plates were sprayed with $75 \%(\mathrm{v} / \mathrm{v}) \mathrm{H}_{2} \mathrm{SO}_{4}$ and were heated at $180^{\circ} \mathrm{C}$ for $30 \mathrm{~min}$. Charred areas were scraped into boiling tubes and $\mathbf{P}$ was determined by a modification of the method of Marinetti (1962), by using a mixture of $70 \%(w / v)$ perchloric acid and concentrated $\mathrm{H}_{2} \mathrm{SO}_{4}(1.2 \mathrm{ml})$ as ashing mixture, with appropriate dilution before colour development to maintain a suitable acid concentration. The $P$ content was calculated from standards that showed a linear relationship between extinction and concentration over the range of $0-10 \mu \mathrm{g}$ of $\mathrm{P} /$ digest. The percentage of $P$ in each component was calculated on the basis of total $\mathbf{P}$ recovered. In this modification of the method of Marinetti (1962), a minor decrease in sensitivity was compensated for by the absence of erroneous results caused by inadvertent losses of ashing mixture during digestion in the presence of silica gel (cf. Owens, 1965).

Total $\mathbf{P}$ in acid digests of whole lipid extracts was determined by a modification of the method of Briggs (1922). Total phospholipid was calculated by the equation: $P \times 25.8 \equiv$ phospholipid (Spector, 1956).

Fatty acids and aldehydes of spermatozoal lipids. Neutral lipids and total phospholipids were separated by t.l.c. on silica gel $\mathrm{G}(250 \mu \mathrm{m})$ by using hexanediethyl ether-acetic acid $(80: 20: 1$, by vol.). Individual phospholipids were separated on silica gel $\mathbf{G}$ $(500 \mu \mathrm{m})$ by using chloroform-methanol-water (65:25:4, by vol.). Components were detected by spraying the plates with Rhodamine $6 \mathrm{G}(0.05 \%$ in ethanol) and viewing under u.v. light. Bands of silica gel containing the separated lipids were treated by the procedure of Metcalfe et al. (1966) and the fatty acid methyl esters were dried over anhydrous $\mathrm{Na}_{2} \mathrm{SO}_{4}$ and stored in hexane at $4^{\circ} \mathrm{C}$. Where lipids contained plasmalogenic aldehydes, the acids and aldehydes were separated by saponification as described by Payne \& Masters (1970) followed by methylation of the separated components.

The resultant methyl esters and dimethyl acetals were analysed by g.1.c. at $190^{\circ} \mathrm{C}$ with $17 \%(\mathrm{w} / \mathrm{w})$ diethylene glycol succinate and $20 \%(\mathrm{w} / \mathrm{w})$ silicone SE30 as stationary phases on Gas Chrom Q 80-100 mesh (Applied Science Laboratories). Calculation was based on the relative peak area (James, 1960) and peaks were identified by comparison of the retention times with pure standards or with published values (Hofstetter et al., 1965). Considerable care was taken in preparing and handling the dimethyl acetals, and all-glass chromatographic system was used to prevent any anomalous breakdown. Diglyceride concentrations were calculated from gas-chromatographic analyses of their fatty acid methyl esters by using methyl margarate as an internal standard.

\section{Results}

\section{General fatty acid incorporation}

More than $95 \%$ of the radioactive label that was added initially was recovered in lipid extracts with incubation periods of up to $2 \mathrm{~h}$. That small amounts of 
Table 1. Percentage distribution of ${ }^{14} \mathrm{C}$ in lipid extracts after incubation of bovine semen with labelled fatty acids for $2 h$

Spermatozoa and seminal plasma fractions were separated by centrifugation at $450 \mathrm{~g}$ for $20 \mathrm{~min}$, then the lipids were extracted as described in the text. Calculations are based on the amount of label recovered in each fraction expressed as a percentage of the total label recovered in both fractions.

$\begin{array}{lcc}\text { Fatty acid } & \text { Spermatozoa } & \text { Seminal Plasma } \\ \text { Myristic } & 82.9 & 17.1 \\ \text { Palmitic } & 86.8 & 13.2 \\ \text { Stearic } & 23.3 & 76.7 \\ \text { Oleic } & 78.8 & 21.2 \\ \text { Linoleic } & 68.9 & 31.1\end{array}$

added fatty acid were oxidized was suggested by the slight decline in recovery of the initial radioactivity with time.

Spermatozoa and seminal plasma were separated after $2 \mathrm{~h}$ of incubation, and the amount of label in each fraction was measured (Table 1). The label in the seminal plasma at this time was predominantly in free fatty acid with trace incorporation into other lipids. As the pattern of this incorporation was similar to that of the spermatozoal lipids, these minor concentrations were interpreted as resulting from contamination of the plasma with a few cells. All fatty acids except stearic acid were actively incorporated into spermatozoa; of those studied palmitic acid was utilized to the greatest extent.

Within lipid extracts, cholesteryl esters and triglycerides contained no demonstrable radioactivity.

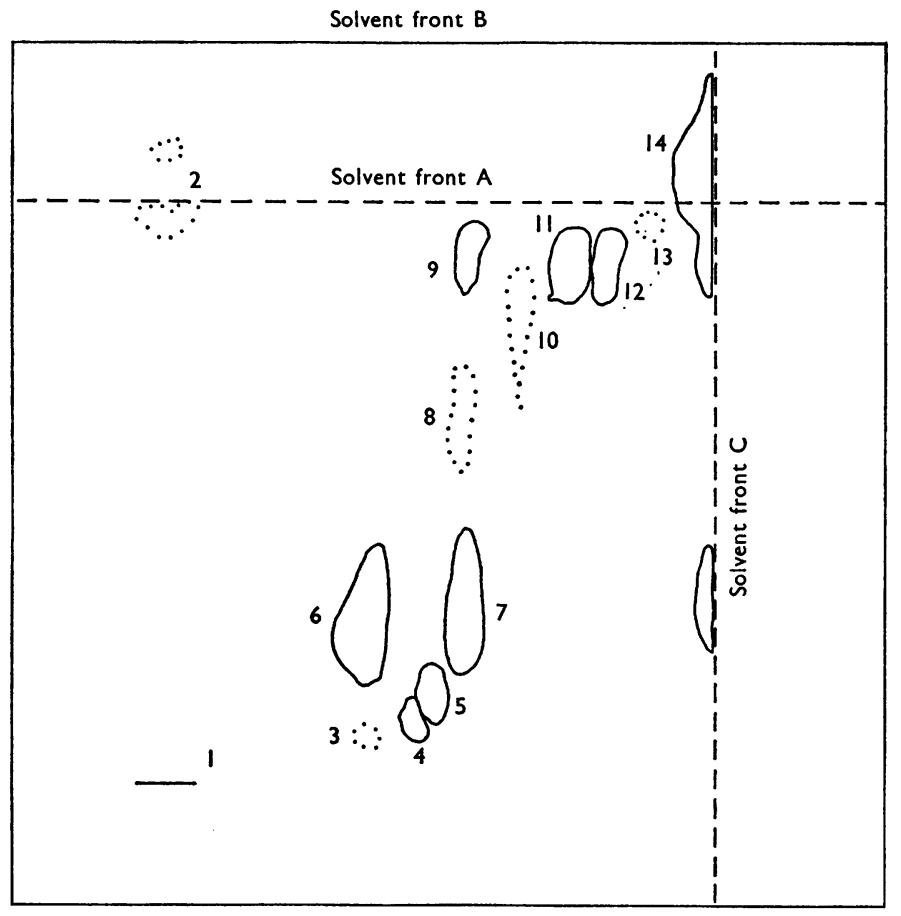

Fig. 1. Two-dimensional thin-layer chromatogram of bovine spermatozoal lipids

First dimension solvents; A, chloroform-methanol-water-acetic acid (65:43:3:1, by vol.); B, hexane-diethyl ether-acetic acid (80:20:1, by vol.). After exposure of the lipid track to $\mathrm{HCl}$ fumes the plate was developed in the second dimension solvent; $C$, chloroform-methanol-water $(65: 35: 8$, by vol.). Plates were sprayed with $75 \%$ (v/v) $\mathrm{H}_{2} \mathrm{SO}_{4}$ and charred. The areas are: 1, origin (lipoprotein); 2, unidentified component; 3, lysophosphatidylcholine; 4 and 5, sphingomyelin; 6, (2-acyl) lysophosphatidylcholine (hydrolysed choline plasmalogen); 7, phosphatidylcholine; 8, phosphatidylserine; 9, (2-acyl) lysophosphatidylethanolamine (hydrolysed ethanolamine plasmalogen); 10, phosphatidylinositol; 11, phosphatidylethanolamine; 12, cerebrosides; 13, cardiolipin and phosphatidic acid; 14, neutral lipid. 


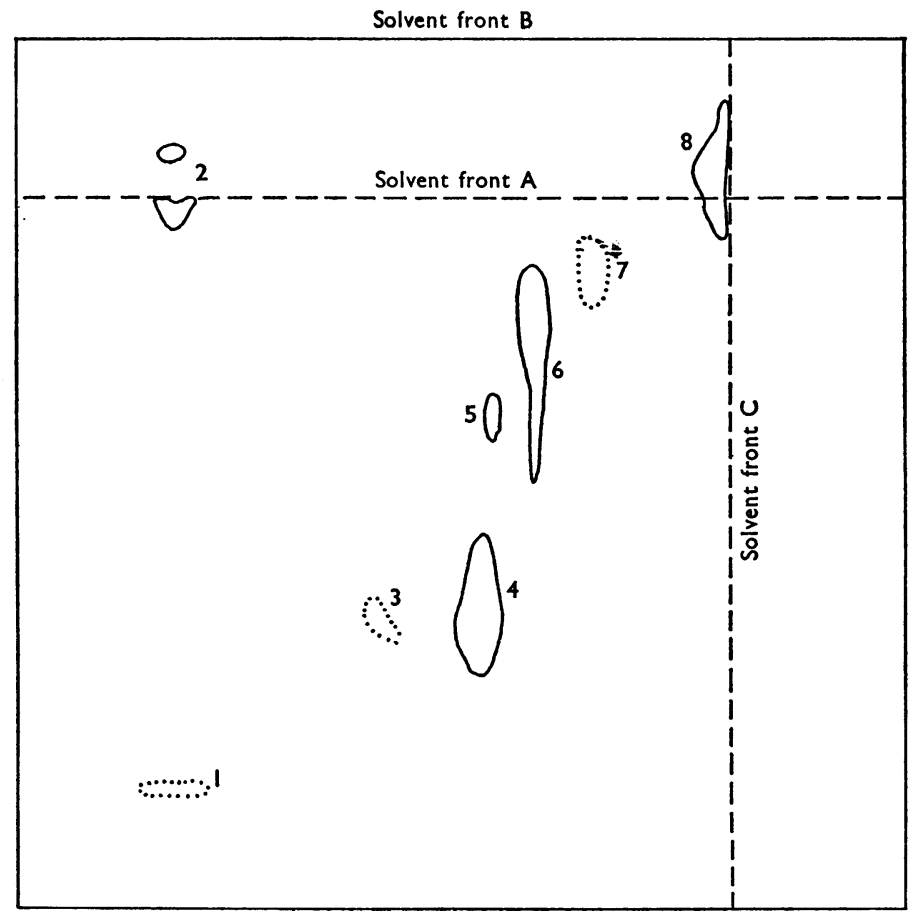

Fig. 2. Radioautogram of bovine spermatozoal lipids after incubation of spermatozoa with $\left[U-{ }^{14} \mathrm{C}\right]$ palmitic acid for $2 h$

Two-dimensional t.l.c. was performed as described in Fig. 1. Areas of radioactivity: 1, origin (lipoprotein); 2, unidentified component; 3, (2-acyl) lysophosphatidylcholine (hydrolysed choline plasmalogen); 4, phosphatidylcholine; 5 , phosphatidylserine; 6 , phosphatidylinositol; 7, phosphatidylethanolamine; 8, neutral lipid.

Low radioactivities were demonstrable in monoglycerides also, but were not estimated because of the difficulty of resolving this component from the total phospholipids. The radioactivity of the free fatty acid pool was also not measured because, in general, unincorporated label was not removed before lipid extraction.

A typical thin-layer chromatogram of bovine spermatozoal phospholipids and a resulting radioautogram after incubation of spermatozoa with labelled fatty acid are shown in Figs. 1 and 2 respectively. An origin spot on thin-layer chromatograms contained 8.8 and $12.3 \mu \mathrm{g}$ of $\mathrm{P} / 10^{9}$ spermatozoa in the samples of lipid extract analysed, and was thought to be proteolipid (Scott \& Dawson, 1968). This component, together with an unidentified component $\left(0.1\right.$ and $0.3 \mu \mathrm{g}$ of $\mathrm{P} / 10^{9}$ spermatozoa in lipid extracts analysed), showed marked but variable incorporation of labelled fatty acid. Sphingomyelin, which characteristically migrated as two discrete components (cf. Rouser et al., 1963), and lysophosphatidylcholine showed no demonstrableactivity.

The specific radioactivities of incorporation of exogenous fatty acids into metabolically active lipids of bovine spermatozoa are presented in Tables 2-6, with calculations based on the means of lipid concentrations in extracts analysed. A preliminary experiment with palmitic acid had shown that incorporation of label was independent of substrate concentration over the present range of fatty acid concentrations (0.6-33.3 nmol/incubation). Assuming that this relationship was true for all acids studied, results have also been expressed in terms of the specific radioactive labelling of sperm lipids to clarify differences in metabolism between the various substrates. The results are not absolute, as insufficient material was available for statistical analysis, but they do represent the reproducible incorporation of fatty acids into spermatozoal lipids under the conditions used.

\section{Incorporation into diglycerides}

The sperm extracts analysed contained 1,2diglycerides at concentrations of 127.4 and $125.7 \mathrm{nmol}$ of glycerol $/ 10^{9}$ spermatozoa and 1,3-diglycerides at 
Table 2. Incorporation of myristic acid into the lipids of bovine spermatozoa

Semen $(1 \mathrm{ml})$ was incubated in air at $32^{\circ} \mathrm{C}$ for various time-intervals with $1 \mathrm{ml}$ of Krebs-Ringer phosphate buffer (pH 7.4) containing $3 \%(\mathrm{w} / \mathrm{v})$ bovine serum albumin, $0.1 \mathrm{ml}$ of $4 \%(\mathrm{w} / \mathrm{v})$ fructose, $0.1 \mathrm{ml}(0.5 \mu \mathrm{Ci})$ of labelled fatty acid in the phosphate-serum albumin buffer and 300 units of penicillin. The specific radioactivity of the $\left[1-{ }^{14} \mathrm{C}\right]-$ myristic acid was $15 \mu \mathrm{Ci} / \mu \mathrm{mol}$. Results are expressed as nmol of fatty acid (and $\mu \mathrm{Ci}$ of ${ }^{14} \mathrm{C}$ ) incorporated $/ \mu \mathrm{mol}$ of glycerol and $\mathbf{P}$ for diglycerides and phospholipids respectively. Values in parentheses are the specific radioactivities.

\begin{tabular}{|c|c|c|c|c|c|}
\hline \multirow{2}{*}{$\begin{array}{l}\text { Lipid fraction } \\
\text { Time }\end{array}$} & \multicolumn{5}{|c|}{ Fatty acid (radioactivity) incorporated } \\
\hline & $2 \mathrm{~min}$ & $15 \mathrm{~min}$ & $30 \min$ & $60 \min$ & $120 \mathrm{~min}$ \\
\hline 1,3-Diglycerides & $\begin{array}{c}6.929 \\
(0.094)\end{array}$ & $\begin{array}{l}49.083 \\
(0.663)\end{array}$ & $\begin{array}{r}116.352 \\
(1.572)\end{array}$ & $\begin{array}{r}153.376 \\
(2.073)\end{array}$ & $\begin{array}{r}174.269 \\
(2.355)\end{array}$ \\
\hline 1,2-Diglycerides & $\begin{array}{c}1.051 \\
(0.014)\end{array}$ & $\begin{array}{l}16.468 \\
(0.223)\end{array}$ & $\begin{array}{l}28.765 \\
(0.389)\end{array}$ & $\begin{array}{l}57.093 \\
(0.772)\end{array}$ & $\begin{array}{l}54.559 \\
(0.737)\end{array}$ \\
\hline Total phospholipid & $\begin{array}{c}0.081 \\
(0.001)\end{array}$ & $\begin{array}{c}0.253 \\
(0.003)\end{array}$ & $\begin{array}{l}0.181 \\
(0.002)\end{array}$ & $\begin{array}{c}0.243 \\
(0.003)\end{array}$ & $\begin{array}{c}0.337 \\
(0.005)\end{array}$ \\
\hline Phosphatidylcholine & $\begin{array}{c}0.048 \\
(0.001)\end{array}$ & $\begin{array}{c}0.187 \\
(0.003)\end{array}$ & $\begin{array}{c}0.223 \\
(0.003)\end{array}$ & $\begin{array}{c}0.382 \\
(0.005)\end{array}$ & $\begin{array}{c}0.910 \\
(0.012)\end{array}$ \\
\hline Phosphatidylinositol & $\begin{array}{c}6.651 \\
(0.090)\end{array}$ & $\begin{array}{l}18.657 \\
(0.252)\end{array}$ & $\begin{array}{l}10.771 \\
(0.146)\end{array}$ & $\begin{array}{l}13.761 \\
(0.186)\end{array}$ & $\begin{array}{l}12.172 \\
(0.165)\end{array}$ \\
\hline Phosphatidylserine & & $\begin{array}{c}0.359 \\
(0.005)\end{array}$ & $\begin{array}{c}0.911 \\
(0.012)\end{array}$ & $\begin{array}{c}0.673 \\
(0.009)\end{array}$ & $\begin{array}{r}0.815 \\
(0.011)\end{array}$ \\
\hline
\end{tabular}

Table 3. Incorporation of palmitic acid into the lipids of bovine spermatozoa

The conditions of incubation and expression of results are as in Table 2. The specific radioactivity of the [U-14 C]palmitic acid was $900 \mu \mathrm{Ci} / \mu \mathrm{mol}$.

\begin{tabular}{|c|c|c|c|c|c|}
\hline \multirow{2}{*}{$\begin{array}{l}\text { Lipid fraction } \\
\text { Time }\end{array}$} & \multicolumn{5}{|c|}{ Fatty acid (radioactivity) incorporated } \\
\hline & $2 \min$ & $15 \mathrm{~min}$ & $30 \mathrm{~min}$ & $60 \mathrm{~min}$ & $120 \mathrm{~min}$ \\
\hline 1,3-Diglycerides & $\begin{array}{c}0.117 \\
(0.096)\end{array}$ & $\begin{array}{c}0.440 \\
(0.356)\end{array}$ & $\begin{array}{c}0.806 \\
(0.653)\end{array}$ & $\begin{array}{c}1.437 \\
(1.164)\end{array}$ & $\begin{array}{c}1.803 \\
(1.461)\end{array}$ \\
\hline 1,2-Diglycerides & $\begin{array}{c}0.018 \\
(0.015)\end{array}$ & $\begin{array}{c}0.155 \\
(0.126)\end{array}$ & $\begin{array}{c}0.370 \\
(0.300)\end{array}$ & $\begin{array}{c}0.521 \\
(0.422)\end{array}$ & $\begin{array}{c}0.713 \\
(0.578)\end{array}$ \\
\hline Total phospholipid & $\begin{array}{c}0.001 \\
(0.001)\end{array}$ & $\begin{array}{c}0.005 \\
(0.004)\end{array}$ & $\begin{array}{c}0.008 \\
(0.007)\end{array}$ & $\begin{array}{c}0.014 \\
(0.011)\end{array}$ & $\begin{array}{c}0.017 \\
(0.014)\end{array}$ \\
\hline Phosphatidylcholine & $\begin{array}{c}<0.001 \\
(<0.001)\end{array}$ & $\begin{array}{c}0.006 \\
(0.005)\end{array}$ & $\begin{array}{c}0.012 \\
(0.009)\end{array}$ & $\begin{array}{c}0.030 \\
(0.023)\end{array}$ & $\begin{array}{c}0.052 \\
(0.039)\end{array}$ \\
\hline Phosphatidylinositol & $\begin{array}{c}0.067 \\
(0.050)\end{array}$ & $\begin{array}{c}0.378 \\
(0.284)\end{array}$ & $\begin{array}{c}0.557 \\
(0.418)\end{array}$ & $\begin{array}{c}0.709 \\
(0.532)\end{array}$ & $\begin{array}{c}0.637 \\
(0.478)\end{array}$ \\
\hline Phosphatidylserine & & $\begin{array}{c}0.065 \\
(0.009)\end{array}$ & $\begin{array}{c}0.051 \\
(0.016)\end{array}$ & $\begin{array}{c}0.250 \\
(0.028)\end{array}$ & $\begin{array}{c}0.210 \\
(0.027)\end{array}$ \\
\hline
\end{tabular}

concentrations of 13.7 and $14.3 \mathrm{nmol}$ of glycerol $/ 10^{9}$ spermatozoa. The diglyceride fractions showed comparatively high incorporation of substrates with marked radioactivity being evident within 2 min of the commencement of incubation, particularly in the 1,3-diglycerides. Compared with the 1,2-diglycerides, this component showed a high specificity for saturated substrates, particularly palmitic and myristic acids. This factor, together with the differences in the rates of incorporation of individual fatty acids into each diglyceride fraction, suggests that there are major differences in their metabolism within spermatozoa. It was notable that, in general, whereas fatty acid incorporation in diglycerides increased with time, maximum uptake of myristic acid into 1,2-diglycerides was observed after $1 \mathrm{~h}$. Associated with this was the fact that, on the basis of the specific radioactivity results, both diglycerides utilized myristic acid to the greatest extent. As these lipids contained very high proportions of myristic acid (Table 7), this 
Table 4. Incorporation of stearic acid into the lipids of bovine spermatozoa

The conditions of incubation and expression of results are as in Table 2 . The specific radioactivity of the $\left[1-{ }^{14} \mathrm{C}\right]-$ stearic acid was $48 \mu \mathrm{Ci} / \mu \mathrm{mol}$.

\begin{tabular}{|c|c|c|c|c|c|}
\hline \multirow{2}{*}{$\begin{array}{r}\text { Lipid fraction } \\
\text { Time }\end{array}$} & \multicolumn{5}{|c|}{ Fatty acid (radioactivity) incorporated } \\
\hline & $2 \mathrm{~min}$ & $15 \mathrm{~min}$ & $30 \mathrm{~min}$ & $60 \mathrm{~min}$ & $120 \mathrm{~min}$ \\
\hline 1,3-Diglycerides & $\begin{array}{c}0.435 \\
(0.019)\end{array}$ & $\begin{array}{c}1.571 \\
(0.068)\end{array}$ & $\begin{array}{c}3.071 \\
(0.133)\end{array}$ & $\begin{array}{l}3.638 \\
(0.158)\end{array}$ & $\begin{array}{c}5.241 \\
(0.227)\end{array}$ \\
\hline 1,2-Diglycerides & $\begin{array}{c}0.133 \\
(0.006)\end{array}$ & $\begin{array}{c}0.555 \\
(0.024)\end{array}$ & $\begin{array}{c}0.946 \\
(0.041)\end{array}$ & $\begin{array}{l}2.206 \\
(0.096)\end{array}$ & $\begin{array}{c}4.328 \\
(0.187)\end{array}$ \\
\hline Total phospholipids & $\begin{array}{c}0.030 \\
(0.001)\end{array}$ & $\begin{array}{c}0.065 \\
(0.003)\end{array}$ & $\begin{array}{c}0.085 \\
(0.003)\end{array}$ & $\begin{array}{c}0.115 \\
(0.005)\end{array}$ & $\begin{array}{c}0.247 \\
(0.010)\end{array}$ \\
\hline $\begin{array}{l}\text { Cardiolipin/ } \\
\text { phosphatidic acid }\end{array}$ & $\begin{array}{c}0.519 \\
(0.022)\end{array}$ & $\begin{array}{c}0.332 \\
(0.014)\end{array}$ & $\begin{array}{c}0.174 \\
(0.008)\end{array}$ & $\begin{array}{c}0.335 \\
(0.015)\end{array}$ & $\begin{array}{c}0.352 \\
(0.015)\end{array}$ \\
\hline $\begin{array}{l}\text { Phosphatidyl- } \\
\text { ethanolamine }\end{array}$ & $\begin{array}{c}0.157 \\
(0.007)\end{array}$ & $\begin{array}{l}0.267 \\
(0.012)\end{array}$ & $\begin{array}{c}0.241 \\
(0.010)\end{array}$ & $\begin{array}{c}0.246 \\
(0.011)\end{array}$ & $\begin{array}{c}0.254 \\
(0.011)\end{array}$ \\
\hline Phosphatidylcholine & & $\begin{array}{c}0.035 \\
(0.002)\end{array}$ & $\begin{array}{c}0.074 \\
(0.003)\end{array}$ & $\begin{array}{c}0.106 \\
(0.005)\end{array}$ & $\begin{array}{l}0.166 \\
(0.007)\end{array}$ \\
\hline Phosphatidylinositol & $\begin{array}{c}1.182 \\
(0.051)\end{array}$ & $\begin{array}{c}2.438 \\
(0.106)\end{array}$ & $\begin{array}{r}3.464 \\
(0.150)\end{array}$ & $\begin{array}{c}5.468 \\
(0.237)\end{array}$ & $\begin{array}{l}14.675 \\
(0.636)\end{array}$ \\
\hline Phosphatidylserine & & $\begin{array}{c}0.354 \\
(0.015)\end{array}$ & $\begin{array}{l}0.750 \\
(0.019)\end{array}$ & $\begin{array}{l}0.340 \\
(0.015)\end{array}$ & $\begin{array}{c}0.760 \\
(0.018)\end{array}$ \\
\hline
\end{tabular}

Table 5. Incorporation of oleic acid into the lipids of bovine spermatozoa

The conditions of incubation and expression of results are as in Table 2 . The specific radioactivity of the $\left[1-{ }^{14} \mathrm{C}\right]-$ oleic acid was $58 \mu \mathrm{Ci} / \mu \mathrm{mol}$.

\begin{tabular}{|c|c|c|c|c|c|}
\hline \multirow{2}{*}{$\begin{array}{r}\text { Lipid fraction } \\
\text { Time }\end{array}$} & \multicolumn{5}{|c|}{ Fatty acid (radioactivity) incorporated } \\
\hline & $2 \mathrm{~min}$ & $15 \mathrm{~min}$ & $30 \mathrm{~min}$ & $60 \mathrm{~min}$ & $120 \mathrm{~min}$ \\
\hline 1,2-Diglycerides & $\begin{array}{c}0.897 \\
(0.047)\end{array}$ & $\begin{array}{c}4.568 \\
(0.239)\end{array}$ & $\begin{array}{c}8.391 \\
(0.440)\end{array}$ & $\begin{array}{c}9.462 \\
(0.496)\end{array}$ & $\begin{array}{l}12.284 \\
(0.643)\end{array}$ \\
\hline 1,3-Diglycerides & $\begin{array}{c}0.292 \\
(0.015)\end{array}$ & $\begin{array}{c}3.389 \\
(0.178)\end{array}$ & $\begin{array}{r}6.536 \\
(0.342)\end{array}$ & $\begin{array}{l}10.523 \\
(0.551)\end{array}$ & $\begin{array}{l}14.577 \\
(0.764)\end{array}$ \\
\hline Total phospholipid & $\begin{array}{c}0.017 \\
(0.001)\end{array}$ & $\begin{array}{c}0.063 \\
(0.003)\end{array}$ & $\begin{array}{c}0.054 \\
(0.003)\end{array}$ & $\begin{array}{c}0.071 \\
(0.004)\end{array}$ & $\begin{array}{c}0.179 \\
(0.009)\end{array}$ \\
\hline Phosphatidylcholine & $\begin{array}{c}0.007 \\
(<0.001)\end{array}$ & $\begin{array}{c}0.031 \\
(0.002)\end{array}$ & $\begin{array}{c}0.068 \\
(0.004)\end{array}$ & $\begin{array}{c}0.185 \\
(0.010)\end{array}$ & $\begin{array}{c}0.514 \\
(0.027)\end{array}$ \\
\hline Phosphatidylinositol & $\begin{array}{c}0.367 \\
(0.019)\end{array}$ & $\begin{array}{c}4.092 \\
(0.214)\end{array}$ & $\begin{array}{c}3.080 \\
(0.161)\end{array}$ & $\begin{array}{c}2.000 \\
(0.105)\end{array}$ & $\begin{array}{c}5.496 \\
(0.288)\end{array}$ \\
\hline Phosphatidylserine & $\begin{array}{l}0.176 \\
(0.009)\end{array}$ & & $\begin{array}{c}0.423 \\
(0.022)\end{array}$ & $\begin{array}{c}0.543 \\
(0.028)\end{array}$ & $\begin{array}{c}0.203 \\
(0.011)\end{array}$ \\
\hline
\end{tabular}

composition might be considered as a possible explanation for the active incorporation of this acid, whereas the lesser utilization of palmitic acid and the relatively poor metabolism of stearic acid may be related to the relative endogenous amounts of these acids in each diglyceride fraction. Unsaturated fatty acids however, were more actively metabolized by 1,2-diglycerides than was palmitic acid; and this observation would seem to suggest that fatty acid metabolism is not directly related to the overall fatty acid composition of endogenous sperm lipids in the case of the unsaturated components.

\section{Incorporation into phospholipids}

The comparatively low radioactivity of the total phospholipids was a reflection of the large pool of relatively inert plasmalogenic phospholipids in 
Table 6. Incorporation of linoleic acid into the lipids of bovine spermatozoa

The conditions of incubation and expression of results are as in Table 2 . The specific radioactivity of the $\left[1-{ }^{14} \mathrm{C}\right]$ linoleic acid was $53 \mu \mathrm{Ci} / \mu \mathrm{mol}$.

\begin{tabular}{|c|c|c|c|c|c|}
\hline \multirow{2}{*}{$\begin{array}{l}\text { Lipid fraction } \\
\text { Time }\end{array}$} & \multicolumn{5}{|c|}{ Fatty acid (radioactivity) incorporated } \\
\hline & $2 \mathrm{~min}$ & $15 \mathrm{~min}$ & $30 \mathrm{~min}$ & $60 \mathrm{~min}$ & $120 \mathrm{~min}$ \\
\hline 1,3-Diglycerides & $\begin{array}{l}1.441 \\
(0.069)\end{array}$ & $\begin{array}{c}4.715 \\
(0.226)\end{array}$ & $\begin{array}{c}7.705 \\
(0.369)\end{array}$ & $\begin{array}{c}9.871 \\
(0.473)\end{array}$ & $\begin{array}{l}13.280 \\
(0.636)\end{array}$ \\
\hline 1,2-Diglycerides & $\begin{array}{c}0.647 \\
(0.031)\end{array}$ & $\begin{array}{r}3.069 \\
(0.147)\end{array}$ & $\begin{array}{l}6.018 \\
(0.288)\end{array}$ & $\begin{array}{l}10.779 \\
(0.517)\end{array}$ & $\begin{array}{l}14.096 \\
(0.675)\end{array}$ \\
\hline Total phospholipid & $\begin{array}{c}0.029 \\
(0.001)\end{array}$ & $\begin{array}{c}0.059 \\
(0.003)\end{array}$ & $\begin{array}{c}0.101 \\
(0.005)\end{array}$ & $\begin{array}{c}0.208 \\
(0.010)\end{array}$ & $\begin{array}{c}0.264 \\
(0.013)\end{array}$ \\
\hline $\begin{array}{l}\text { Cardiolipin/ } \\
\text { phosphatidic acid }\end{array}$ & $\begin{array}{c}0.581 \\
(0.028)\end{array}$ & $\begin{array}{c}0.563 \\
(0.027)\end{array}$ & $\begin{array}{c}0.790 \\
(0.038)\end{array}$ & $\begin{array}{c}0.567 \\
(0.027)\end{array}$ & $\begin{array}{c}0.385 \\
(0.018)\end{array}$ \\
\hline $\begin{array}{l}\text { Phosphatidyl- } \\
\text { ethanolamine }\end{array}$ & $\begin{array}{c}0.115 \\
(0.006)\end{array}$ & $\begin{array}{c}0.148 \\
(0.007)\end{array}$ & $\begin{array}{c}0.144 \\
(0.007)\end{array}$ & $\begin{array}{c}0.106 \\
(0.005)\end{array}$ & $\begin{array}{c}0.260 \\
(0.012)\end{array}$ \\
\hline Phosphatidylcholine & & & $\begin{array}{c}0.140 \\
(0.007)\end{array}$ & $\begin{array}{c}0.492 \\
(0.024)\end{array}$ & $\begin{array}{c}0.663 \\
(0.032)\end{array}$ \\
\hline Phosphatidylinositol & $\begin{array}{c}1.202 \\
(0.058)\end{array}$ & $\begin{array}{c}3.278 \\
(0.157)\end{array}$ & $\begin{array}{c}4.143 \\
(0.199)\end{array}$ & $\begin{array}{c}6.468 \\
(0.310)\end{array}$ & $\begin{array}{c}5.690 \\
(0.273)\end{array}$ \\
\hline Phosphatidylserine & & $\begin{array}{c}0.204 \\
(0.010)\end{array}$ & $\begin{array}{c}0.365 \\
(0.018)\end{array}$ & $\begin{array}{c}0.859 \\
(0.041)\end{array}$ & $\begin{array}{c}0.819 \\
(0.039)\end{array}$ \\
\hline
\end{tabular}

Table 7. Fatty acid composition of lipids extracted from bovine spermatozoa

Fatty acid composition (\%)

\begin{tabular}{|c|c|c|c|c|c|c|c|}
\hline $\begin{array}{l}\text { Fatty } \\
\text { acid }\end{array}$ & $\begin{array}{l}\text { 1,3-Digly- } \\
\text { cerides }\end{array}$ & $\begin{array}{l}\text { 1,2-Digly- } \\
\text { cerides }\end{array}$ & $\begin{array}{c}\text { Total } \\
\text { phospho- } \\
\text { lipid }\end{array}$ & Cardiolipin & $\begin{array}{l}\text { Ethanolamine } \\
\text { phospho- } \\
\text { glycerides }\end{array}$ & $\begin{array}{l}\text { Choline } \\
\text { phospho- } \\
\text { glycerides }\end{array}$ & $\begin{array}{c}\text { Phosphatidyl } \\
\text { inositol }\end{array}$ \\
\hline 14:0 & 58.4 & 69.6 & 3.9 & 5.4 & 5.2 & 2.0 & 14.4 \\
\hline $16: 0$ & 23.3 & 22.7 & 16.5 & 11.8 & 15.2 & 9.5 & 43.7 \\
\hline 18:0 & 3.9 & 3.8 & 6.0 & 0.3 & 11.1 & 1.2 & 7.3 \\
\hline $18: 1, n-9$ & 13.6 & 3.2 & 3.9 & 23.6 & 5.5 & 1.2 & 5.6 \\
\hline $18: 2, n-6$ & 0.8 & 0.7 & 3.0 & 50.8 & 0.8 & 0.3 & 0.9 \\
\hline $18: 3, n-3$ & & Trace & 0.3 & Trace & 1.0 & & Trace \\
\hline $20: 3, n-6$ & & Trace & 0.4 & 2.3 & & & \\
\hline $20: 4, n-6$ & & Trace & 3.3 & 5.4 & 18.8 & 0.7 & 1.5 \\
\hline $22: 5, n-6$ & & & 6.9 & Trace & 4.7 & 6.2 & 0.4 \\
\hline $22: 6, n-3$ & & & 54.5 & Trace & 37.8 & 78.8 & 24.9 \\
\hline
\end{tabular}

bovine sperm. Though only trace amounts of radioactivity were observed in these lipids, some specificity was observed. Palmitic acid, but not stearic acid, was incorporated into choline plasmalogen whereas with ethanolamine plasmalogen these incorporations were reversed. Both phospholipids incorporated linoleic acid and trace labelling of choline plasmalogen was also evident with myristic and oleic acids. The plasmalogens also differed in the rate of incorporation of fatty acids, radioactivity in ethanolamine plasmalogen being evident after 2 min of incubation with stearic acid whereas the earliest appearance of label in choline plasmalogen was after 30 min of incubation with palmitic acid.

Phosphatidylcholine, the major diacyl phospholipid of sperm, showed minor metabolic activity with a general increase in incorporation of fatty acid with time. Myristic, palmitic and oleic acids were rapidly incorporated into phosphatidylcholine, but there was a delay in the incorporation of stearic and linoleic acids. Phosphatidylcholine utilized palmitic acid to the greatest extent with linoleic and oleic acids also being actively incorporated after $2 \mathrm{~h}$. By comparison, myristic and stearic acids were poorly incorporated.

Vol. 127 
Phosphatidylethanolamine and the cardiolipin and phosphatidic acid fractions showed minor activity with all substrates except stearic and linoleic acids. Whereas inital incorporation of these acids into both components was relatively active, subsequent incubations showed considerable variability in uptake. It would appear that the conditions of incubation may greatly influence the metabolism of these components, as other workers have reported greater radioactivity in phosphatidylethanolamine (Mills \& Scott, 1968; Payne \& Masters, 1970), and, in the present study, if conditions were changed to form a more dilute medium with buffer, there was an apparent increase in the radioactivity of phosphatidylethanolamine.

The most notable aspect of phospholipid metabolism in sperm was the very active incorporation of all fatty acids into phosphatidylinositol. After $2 \mathrm{~min}$, fatty acid uptake exceeded that into the 1,2-diglyceride fraction and myristic acid incorporation was comparable with that into the 1,3-diglycerides. This acid was maximally incorporated into phosphatidylinositol after $15 \mathrm{~min}$, whereas with oleic acid, after an initial peak of activity at this time, further incorporation was observed after $2 \mathrm{~h}$. More stearic acid was incorporated into phosphatidylinositol than into any of the other lipid fractions, with an increased amount of incorporation at all incubation periods. With palmitic and linoleic acids, maximum incorporation into phosphatidylinositol was established after $1 \mathrm{~h}$.

Marked but variable radioactivity was incorporated into phosphatidylserine with an apparent delay in the metabolism of this minor phospholipid with all substrates except oleic acid.

As with the diglyceride fractions, the active utilization of unsaturated acids by individual phospholipids suggested that metabolic activity may not be directly related to their overall fatty acid composition, in this case.

\section{Fatty acids of sperm lipids}

The percentage composition of fatty acids present in various lipid classes of bovine spermatozoa is presented in Table 7. Acids of shorter chain length than 14 carbon atoms, and the 14 carbon and 16 carbon monoenes were not determined.

The high amounts of myristic acid in the diglyceride fractions are similar to those already reported for these lipids in bovine spermatozoa (Payne \& Masters, 1970). Oleic acid was present in high proportions in the 1,2-diglycerides, but long-chain polyunsaturated fatty acids were found in trace amounts only in the 1,2-diglycerides.

Myristic acid also occurred in significant proportions in the phospholipids, being one of the major components of the phosphatidylinositol fraction. Palmitic acid was the major fatty acid of this phos- pholipid, and the major saturated fatty acid of other phosphoglycerides.

The most prominent feature of the results is the very high proportion of docosahexaenoic acid in the phospholipids. The percentage of this acid in the choline phosphoglycerides suggested that the choline plasmalogen fraction contained this acid almost exclusively; because there was not sufficient material it was not possible to separate the choline and ethanolamine phosphoglycerides into their respective diacyl and alkenyl acyl components for fatty acids analysis.

Arachidonic acid was the second major component of ethanolamine phosphoglycerides, the amount found in the present investigation being about twice that reported by Pursel \& Graham (1967) who also reported higher amounts of linoleic acid in this phospholipid in bovine spermatozoa. The amount of linoleic acid was low in all phospholipids except cardiolipin. The high concentrations of oleic and linoleic acid in cardiolipin were similar to those found in this phospholipid in most tissues, suggesting a common functional role for this mitochondrial lipid.

\section{Plasmalogenic aldehydes in spermatozoa}

Analyses of plasmalogenic aldehydes as their dimethyl acetals showed that palmitaldehyde was the major aldehyde of both choline and ethanolamine plasmalogens, accounting for 85 to $90 \%$ of the aldehydes in each fraction. Stearaldehyde and myristaldehyde were present in about equal proportions. The wide range of aldehyde components noted by Gray (1960) for ram spermatozoa, and Payne \& Masters (1970) for bovine spermatozoa, were not found, and we did not find the relatively high amount of myristaldehyde reported by Pursel \& Graham (1967).

\section{Phospholipid composition of spermatozoa}

Ejaculated spermatozoa from two bulls were analysed for their phospholipid content. Some differences were noted between these analyses and the reported range of concentrations for bovine spermatozoal phospholipids (Table 8). The phosphatidylcholine and phosphatidylethanolamine contents fell below the reported values whereas the concentrations of choline plasmalogen and sphingomyelin exceeded those previously found. The diacyl and alkenyl acyl phosphoglyceride concentrations in bovine spermatozoa reported by Pursel \& Graham (1967), however, were calculated on the basis of the phenylhydrazine method for estimation of plasmalogens, which does not yield results consistent with calculations based on the ratio of aldehydes to fatty acids derived from phospholipids. 
Table 8. Composition of spermatozoa phospholipid

Results are expressed as $\mu \mathrm{g}$ of lipid $/ 10^{9}$ spermatozoa calculated from the $\mathrm{P}$ estimation (lipid $=\mathrm{P} \times 25.8$ ).

\begin{tabular}{|c|c|c|c|}
\hline & Bull 1. & Bull 2. & $\begin{array}{c}\text { Range in bovine } \\
\text { spermatozoa } \\
\text { (Pursel \& Graham, } \\
\text { 1967) }\end{array}$ \\
\hline Total phospholipid & 1464 & 1470 & $921-1791$ \\
\hline Cardiolipin and phosphatidic acid & 23 & 28 & \\
\hline Phosphatidylethanolamine & 97 & 68 & $176-442$ \\
\hline Ethanolamine plasmalogen & 101 & 106 & $45-157$ \\
\hline Phosphatidylcholine & 320 & 291 & $360-624$ \\
\hline Choline plasmalogen & 665 & 665 & $224-514$ \\
\hline Sphingomyelin & 201 & 248 & $56-210$ \\
\hline Phosphatidylinositol & 18 & 13 & \\
\hline Phosphatidylserine & 29 & 22 & \\
\hline Lysophosphatidylcholine & 9 & 21 & \\
\hline
\end{tabular}

\section{Discussion}

In these results, the variability and high specific radioactivities that are features of the incorporation of long-chain fatty acids into phosphatidylinositol contrast markedly with the general incorporation of fatty acids into the major lipids of bovine spermatozoa, and appear to signify a distinct metabolic role for this minor phospholipid. The rapid labelling of phosphatidylinositol in sperm is reminiscent of the induced metabolism of acidic phospholipids that has been reported for other tissues, and which has resulted in considerable speculation about the metabolic significance of this phenomenon in relation to hormonal mechanisms, membrane contraction and transport (Hokin \& Hokin, 1958, 1964; Karnovsky \& Wallach, 1961; Vignais et al., 1964; Scott et al., $1968 a, b)$. Although the relevance of such metabolic activity in sperm remains to be firmly established, an observation of the decreased radioactivity of phosphatidylinositol in a system of decreased motility and coincident with an increase in the radioactivity of other phospholipids appears to denote a possible association between this component and the mechanisms involved in sperm motility (A. R. Neill, unpublished work).

In addition, the present results allow comment on the pathways of lipid metabolism in spermatozoa. The rapid labelling of the 1,2-diglyceride fraction, for example, suggests a relationship between a metabolic pool of this component and phosphatidylinositol. The enzymic degradation of phosphatidylinositol to $\mathrm{D}-\alpha, \beta$-diglyceride and inositol 1-phosphate has been demonstrated in several systems (Dawson, 1959; Kemp et al., 1961; Atherton et al., 1966) and the formation of inositol phosphate from phosphatidylinositol by washed ram spermatozoa indicates that a phosphoinositide inositol-phosphohydrolyase is also present in spermatozoa (Scott \& Dawson, 1968). In the present studies, the initial degree of fatty acid incorporation into phosphatidylinositol exceeded that into the 1,2-diglyceride fraction and is indicative of such a metabolic pathway. Further, the relatively high proportion of myristic acid in phosphatidylinositol indicates a common metabolic fatty acid pool for this component and the diglycerides. As suggested by Scott $e t$ al. (1968b) for thyroid tissue, these factors may be associated with rapid cytostructural changes in the lipoprotein membrane. At later stages of incubation, incorporation into the 1,2-diglycerides generally exceeded that into phosphatidylinositol, indicating that the bulk of the label entering this neutral lipid fraction may have been incorporated via the normal metabolic pathway involving phosphatidic acid intermediates (Kennedy, 1957; Payne \& Masters, 1970). The marked radioactivity in 1,3diglycerides implies that the pathway of acylation of monoglycerides (Clark \& Hübscher, 1961) also plays an active role in fatty acid incorporation in spermatozoa, but differences in the rates of incorporation of label into 1,3- and 1,2-diglycerides suggest differences in metabolism in relation to the positional acylation of monoglycerides.

Although Turner \& Korsh (1962) have shown minor labelling of the glycerol moiety of choline phosphoglycerides from bull sperm after incubation with ${ }^{14} \mathrm{C}$-labelled glycerol and glucose, Dawson (1958) found that $\left.{ }^{32} \mathrm{P}\right] \mathrm{P}_{1}$ was not incorporated into the phospholipids of ram sperm, in vitro. Consequently, it would appear that sperm phospholipid synthesis via the Kennedy pathway (Kennedy, 1957) may not be very active in the presence of glycolysable substrates; and the present results suggest that fatty acids may be incorporated into sperm phospholipids predominantly by means of acyl-CoA phospholipid 
transferase reactions (Lands \& Merkel, 1963). On this basis, the observed incorporation of fatty acids into phosphatidylethanolamine warrants some comment.

Phospholipase A, present in both spermatozoa and seminal plasma, has been shown to have a high degree of specificity for exogenous ethanolamine phosphoglycerides in semen, there being considerable variability in its activity, largely dependent on the seminal plasma components (Scott \& Dawson, 1968). Phosphatidylinositol is also actively degraded, but the enzyme has little activity towards choline phosphoglycerides. Although endogenous phospholipids mainly appear to be shielded from such enzyme activity, the variability in turnover of phosphatidylethanolamine under slightly altered conditions of incubation, and the differing metabolic activities previously observed with this phospholipid (Mills \& Scott, 1968; Payne \& Masters, 1970) may be explainable in terms of phospholipase activity. The irregular metabolism of phosphatidylethanolamine may be indicative of the competitive behaviour of phospholipases and acyl transferases towards a metabolic pool of endogenous ethanolamine phosphoglycerides; the relative incorporation of fatty acids reflecting the specificities of the enzymes involved.

Although such an argument supports the observed higher turnover of choline phosphoglycerides compared with ethanolamine phosphoglycerides, it is not consistent with the active incorporation of fatty acids into phosphatidylinositol. In view of the apparent metabolic importance of this phospholipid in ejaculated spermatozoa, however, it may be subject to protection from phospholipase activity, although the observed decrease in phosphatidylinositol content in ram spermatozoa as they move through the epididymis has been attributed to the action of phospholipase (Scott et al., 1967; Scott \& Dawson, 1968).

The exceptionally large amounts of docosahexaenoic acid found in the phospholipids of bovine spermatozoa are also notable, as they appear to be higher than the previously reported values in any mammalian tissue. The proportions of $(n-6)$ and $(n-3)$ acids present in ejaculated spermatozoa contrast markedly with the relative proportions of these acids present in reproductive tissues. Derivatives of linoleic acid are the major components of bovine and porcine testis (Holman \& Hofstetter, 1965) and also rat testis (Davis et al., 1966). As germ cells account for a major proportion of the volume of testis (Roosen-Runge, 1956), their lipid composition could be expected to be reflected in the lipid pattern of the whole organ, as has been shown in the rat (Davis et al., 1966). Holman \& Hofstetter (1965) have suggested that the very large amounts of $(n-6)$ acids in testicular tissue indicates the functional importance of these compounds in reproductive processes, whereas $(n-3)$ acids, known to have less biological activity than ( $n-6)$ acids (Aaes-Jørgensen, 1961), probably do not play a large role in the lipids of this tissue. The rationale of the present situation, then, may lie in the observed large decrease in phospholipid composition of spermatozoa as they pass through the epididymis (Scott et al., 1967), the components containing the biologically active $(n-6)$ acids being utilized during this maturation process, while those containing $(n-3)$ acids serve to maintain the integrity of ejaculated spermatozoa. Indeed, the high proportion of choline phosphoglycerides in spermatozoa exhibiting low metabolic activity, points to the relative stability of a major pool of bovine spermatozoal lipids, which may well be related to the high proportion of docosahexaenoic acid present. However, the relatively small pool of $(n-6)$ acids in ejaculated spermatozoa would appear to have some metabolic significance as shown by the degree incorporation of linoleic acid in this cell type.

The authors thank the staff of the Artificial Insemination Centre, Department of Primary Industries, Wacol, for the provision of semen, and Mr. R. A. Manning for his technical assistance.

\section{References}

Aaes-Jørgensen, E. (1961) Physiol. Rev. 41, 1

Atherton, R. S., Kemp, P. \& Hawthorne, J. N. (1966) Biochim. Biophys. Acta 125, 409

Briggs, A. P. (1922) J. Biol. Chem. 53, 13

Clark, B. \& Hübscher, G. (1961) Biochem. J. 80, 12P

Davis, J. T., Bridges, R. B. \& Coniglio, J. G. (1966) Biochem. J. 98, 342

Dawson, R. M. C. (1958) Biochem. J. 68, 512

Dawson, R. M. C. (1959) Biochim. Biophys. Acta 33, 68

Flispe, R. J. (1960) J. Dairy Sci. 43, 777

Folch, J., Lees, M. \& Sloane-Stanley, G. H. (1957) J. Biol. Chem. 226, 497

Gray, G. M. (1960) Biochem. J. 77, 82

Hartree, E. F. \& Mann, T. (1961) Biochem. J. 80, 464

Hofstetter, H. H., Sen, N. \& Holman, R. T. (1965) J. Amer. Oil Chem. Soc. 42, 537

Hokin, L. E. \& Hokin, M. R. (1958) J. Biol. Chem. 233, 508

Hokin, L. E. \& Hokin, M. R. (1964) in Metabolism and Physiological Significance of Lipids (Dawson, R. M. C. \& Rhodes, D. N., eds.), p. 423, John Wiley and Sons, London

Holman, R. T. \& Hofstetter, H. H. (1965) J. Amer. Oil Chem. Soc. 42,540

James, A. T. (1960) Methods Biochem. Anal. 8, 1

Karnovsky, M. L. \& Wallach, D. F. H. (1961) J. Biol. Chem. 236, 1895

Kemp, P., Hübscher, G. \& Hawthorne, J. N. (1961) Biochem. J. 79, 193

Kennedy, E. P. (1957) Annu. Rev. Biochem. 26, 119

Lands, W. E. M. \& Merkel, J. (1963) J. Biol. Chem. 238, 398 
Marinetti, G. V. (1962) J. Lipid Res. 3, 1

Metcalfe, L. D., Schmitz, A. A. \& Pelka, J. R. (1966) Anal. Chem. 38, 514

Mills, S. C. \& Scott, T. W. (1968) J. Reprod. Fert. 18, 367

Neill, A. R. \& Masters, C. J. (1971) J. Reprod. Fert. 24, 295

Owens, K. (1965) Biochem. J. 100, 354

Payne, E. \& Masters, C. J. (1969) Comp. Biochem. Physiol. 29, 717

Payne, E. \& Masters, C. J. (1970) Int. J. Biochem. 1, 409

Pursel, V. G. \& Graham, E. F. (1967) J. Reprod. Fert. 14, 203

Roosen-Runge, E. C. (1956) Fert. Steril. 7, 251

Rouser, G., Kritchevsky, G., Heller, D. \& Lieber, E. (1963) J. Amer. Oil Chem. Soc. 40, 425

Scott, T. W. \& Dawson, R. M. C. (1968) Biochem. J. 108, 457
Scott, T. W., Voglmayr, J. K. \& Setchell, B. P. (1967) Biochem.J. 102, 456

Scott, T. W., Hansel, W. \& Donaldson, L. E. (1968a) Biochem. J. 108, 317

Scott, T. W., Mills, S. C. \& Frienkel, N. (1968b) Biochem. $J .109,325$

Spector, W. S. (1956) Handbook of Biological Data, p. 59, W. B. Saunders Co., Philadelphia and London

Turner, C. \& Korsh, G. (1962) Biochemistry 1, 367

Umbreit, W. W., Burris, R. H. \& Stauffer, J. F. (1964) Manometric Techniques, 4th edn., p. 132, Burgess Publishing Co., Minneapolis

Vignais, P. M., Vignais, P. V. \& Lehninger, A. L. (1964) J. Biol. Chem. 239, 2011

Viswanathan, C. V., Phillips, F. \& Lundberg, W. O. (1968) J. Chromatogr. 35, 66 